\title{
Design and Implementation of an Adaptive Virtual Maintenance Training System
}

\author{
Lan Yunkui ${ }^{1, a}$, Li Xingxin ${ }^{1, b}$, and Wang Peng ${ }^{1, c}$ \\ ${ }^{1}$ Army Engineering University of PLA, Shijiazhuang 050003, China \\ aneoman1998@sohu.com, ${ }^{b}$ Ixx1226@sina.com, ${ }^{c}$ wpclever@foxmail.com
}

Keywords: cognitive mechanism; adaptive; virtual maintenance training

Abstract: In order to improve the efficiency of maintenance training and to reduce the cost of maintenance training, this paper has studied the adaptive virtual maintenance training system based on cognitive mechanism. It has also designed the software of the virtual maintenance training system and realized the adaptive virtual maintenance training based on cognitive mechanism, after analyzing and designing the function and interactive process adaptive method of the adaptive virtual maintenance training system.

\section{Introduction}

With the development of technology and modern production, large-scale equipment has became not only more complex in structure, but also more diversified in methods of human-computer interaction, which has greatly increased the difficulty and cost of maintenance. And the level of theoretical knowledge and practical operation of maintenance personnel has also been improved accordingly. Maintenance training can improve the level of maintenance personnel's theoretical knowledge and practical skills ${ }^{[1]}$. The traditional maintenance training method is mainly combined with the physical equipment to carry out related theoretical learning and actual maintenance operation training. This training method is simple and easy to understand and can best simulate real scenes ${ }^{[2]}$. However, because it must be based on the physical characteristics, many shortcomings and problems have also emerged during the maintenance and training of large-scale equipment [3]. In such cases, how to effectively improve the efficiency and reduce the cost of maintenance training has become a major problem in the field of large-scale equipment maintenance.

Virtual maintenance training is a new technology based on virtual reality. Its emergence has effectively solved the problem of traditional maintenance training and has received wide attention. It has become a research hot spot in the field of large-scale equipment maintenance. Nevertheless, the traditional virtual maintenance training system treats all trainees equally, ignoring the individual differences, which only changes the teaching situation but fails to achieve intelligent education ${ }^{[3]}$. In order to realize the requirements of adaptive learning and improve the efficiency and quality of virtual maintenance training, it is necessary to study the adaptive virtual maintenance training technology. Cognition refers to the acquisition of knowledge through a series of psychological 
activities such as the formation of concepts, perceptions, judgments, or imaginations. It corresponds to emotions and wills. Cognitive mechanism refers to the basic processes and laws of cognition ${ }^{[4]}$. The ultimate purpose of maintenance training is to enable the trainees to obtain maintenance knowledge and skills. The cognitive perception of people in the maintenance training environment is an unavoidable problem. Therefore, from the perspective of cognition, it is the fundamental way to improve the effect of maintenance training to study how to design, develop and implement reasonable maintenance training. This thesis has studied the adaptive virtual maintenance training system from the basis of cognitive mechanism.

\section{Functional Design of Adaptive Virtual Maintenance Training System}

The virtual maintenance training system has a complete training function and can support related personnel to complete maintenance training tasks. The system has training functions such as teaching, learning, rehearsals, and assessment: it can support the teaching of training personnel, and can also support the training personnel to monitor, guide, and evaluate the training process; It can support the trainees to study the relevant maintenance theory, and also support the trainees to carry out maintenance operations, task exercises, and skills assessment. Among them, learning and practicing are basic training functions a virtual maintenance training system must have, and assessment and teaching belong to the advanced training functions in this system.

The virtual maintenance training system has four basic components, namely, virtual environment, human-computer interaction, training course system and training monitoring.

As a virtual reality system based on the transfer of maintenance knowledge and skills, the trainees are the main interactions of the virtual maintenance training system and the recipients of maintenance knowledge. The ultimate criterion for evaluating the completion of virtual maintenance training is whether the trainees have acquired the required maintenance knowledge and skills to achieve the training goals.

There must be individual differences in learning ability, knowledge and skills base, learning style habits, etc., and maintenance training has uniform and universal requirements for the training goals of trainees. That is, each trainee must reach the required level of knowledge and skills to be able to pass the training, which requires the virtual maintenance training system providing tailored training programs that are tailored to individual differences.

The adaptive virtual maintenance training system should have the following functions: (1) The training content can be selected according to the trainees' learning ability, knowledge and skills basic test results, and the training plan can be designed; (2) The training content and training plan can be selected and adjusted according to the training level and training situation of the trainees; (3)It can provide targeted training process control strategies according to the trainees' learning style habits; (4) It can monitor the training person's maintenance interaction behavior, and give corrective measures for the error operation; (5) It can evaluate the trainees' virtual training situation and adjust the training process control strategy according to the training situation.

Through the learning ability test, the learning style test obtains the learning characteristics of the trainees, and matches the corresponding individualized training process control strategy. According to the equipment maintenance training content system that needs to be completed, it is necessary to conduct equipment maintenance knowledge and skills level testing, to obtain the subject's current maintenance knowledge and skills level, to compare with the maintenance training goals, to get the trainees' individualized maintenance training content, to combine the trainees' learning characteristics, and to form a personalized maintenance training plan.

Through the training process data recording, test results recording to obtain the training data, the system will dynamically adjust the training content, training plan, training process control strategy 
model based on training data, to achieve self-optimization of the adaptive model. The adaptive model library will guide the personalized training process control strategy, the generation of personalized maintenance training content maintenance training plan, forming a self-optimized closed loop of the adaptive system virtual maintenance training system. The functional framework and functional relationship of the adaptive virtual maintenance training system are displayed in Figure 1.

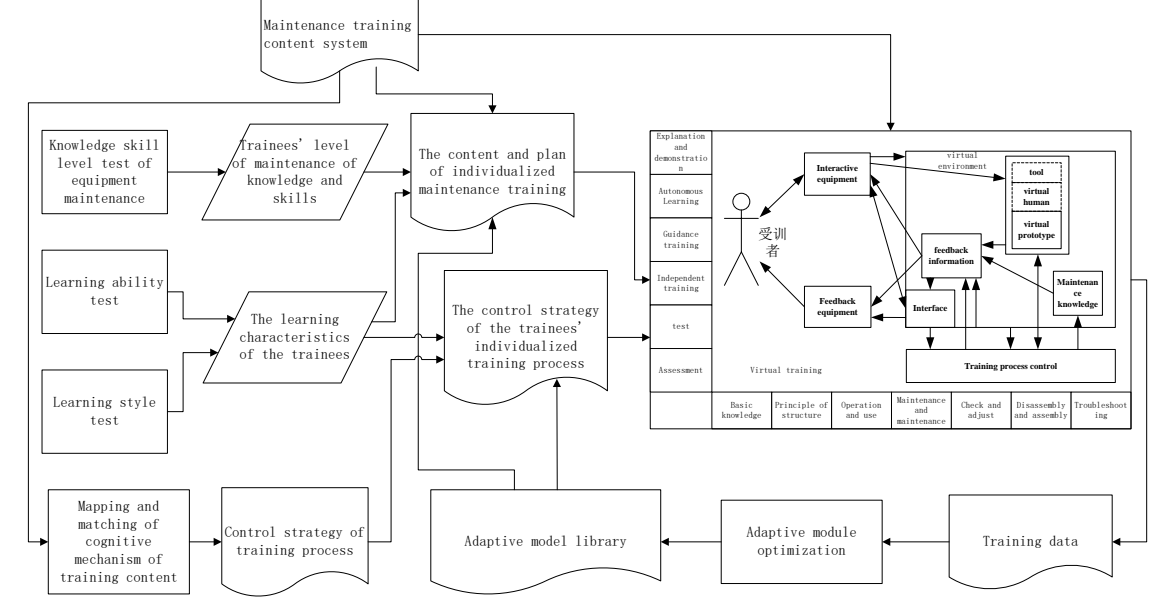

Fig. 1 functional framework and functional diagram of adaptive virtual maintenance training system

\section{Adaptive Design of Virtual Maintenance Training}

\subsection{Type of Learning Feature}

Learning characteristics of the trainees mainly include two aspects: learning ability and learning style.

(1) Learning ability

Learning ability can be divided into seven categories: ability to remember, ability to classify, ability to understand, ability to solve deterministic problems, ability to predict, ability to introspect, and heuristic ability. Each type of ability distinguishes between two ranges.

(2) Learning style

Learning style of the trainees can be divided into four categories. Trainees' learning styles are divided into four categories: contemplative, intuitive, verbal, and comprehensive. Different learning styles determine the learning style of the trainees in the learning process. Meditation learners have a certain amount of time to sum up their experiences before each next stage of teaching. Intuitive learners are more sensitive to the relationship among different things and should reduce repetition and excessive memory; Speech learners should have more words or sounds to stimulate memory and gain deeper understanding; Comprehensive learners should comb the global knowledge when learning, and it is more suitable for them to have a combined memory with the whole concept and the context content.

\subsection{Training Content Adaptive Matching}

The individualized matching of training content adopts the common assessment pass and supplement training methods in maintenance training, that is, for each training subject, the training designer provides the method of knowledge ability test and the criterion of completion. During the maintenance knowledge and skills level test, Traversal tests are conducted for all training subjects, 
and three results of "no training", "restorative training" as well as "complete training" are given according to the characteristics of maintenance training subjects. Training subjects that do not require training judgment results have also been provided, and the first pass date will be automatically recorded. The next judgment will be based on the forgetting parameters in the memory rule and the first pass time to determine whether "restorative training" or "complete training" is required. For training subjects that have reached the trigger condition for restorative training, the system will automatically push the training content.

\subsection{Adaptive design in the Process of Presentation}

(1) According to the learning characteristics of different trainees, they choose to whether explain automatically or not. The explanation process is carried out in a virtual training environment. Trainees can perform common interaction operations such as pause, playback, fast-forward, retreat, selection of play progress, and repetition. Monitor the number of interactive operations such as suspension, playback, fast-forward, retreat, selection of play progress, and repetition, record the learning time, classify and record according to the learning characteristics of trainees, and dynamically adjust the meta-data value of the typical learning time of the topic. After explanation, according to the trainees' learning characteristics, the system automatically pushes the repetition of the explanation or enters the assessment. The trainees can also refuse the push and make their own choices.

(2) Monitor and record the trainees' position movement and observation perspectives in the virtual scene, classify and record according to the trainees' learning characteristics, and automatically push the default position and perspective.

(3) When the microscopic structure, action details, and principle details are being explained, they can be automatically enlarged and moved to the place touched by the trainees. The virtual prototype can be controlled by the handle to perform multi-angle rotation observations (At that time, the system records each observation and provides analysis.). The next step and repeat button is available and the trainees can select the next step when they want the next explanation and they can press the repeat button if they need to listen again (the system records the number of repeats at the same time). The system automatically pushes the repetition times according to the learning characteristics of the trainees.

(4) According to the learning characteristics of the trainees, the learning efficiency of the trainees is predicted, and the targeted tips and help functions are given. By default, the system provides play/change background music, questions, reminder animations (water spray, dog release, shark release, etc.), warning text, lock screens, etc., to improve trainees 'attention and learning efficiency. The trigger conditions are to monitor the offset time of the virtual scene angle, the static time trigger of the monitoring handle, and the trigger time at a predetermined interval according to the trainees 'learning style. Trainees are given different initial values according to different learning characteristics and adjusted dynamically according to the training history.

\subsection{Adaptive Design in Training}

(1) Monitor and record the trainees' position movement, operating position, and observation perspective in the virtual scene, classify and record according to the trainees' learning characteristics, and automatically push the default operating position and viewing angle.

(2) Make correct judgments on each interaction and correct the wrong operation in time, preventing the formation of wrong operation habits. The system classifies and records error 
operations according to learning characteristics of the trainees, and automatically pushes the prompt information for steps with a high error rate.

(3) If there is a serious error in operation, the system should issue an alarm and alert the trainees to give a virtual simulation animation of the consequences of the error. The system records the serious error operation and gives the trigger threshold value according to the trainees' learning characteristics, judging whether to force the suspension operation to re-enter the explanation learning mode.

(4) In the process of dismantling and assembling, the small parts such as screws should be automatically (or reminded to) placed in the receiving box of the parts. The system automatically judges whether the parts are placed in order according to the placement position of the large parts, and gives the trigger threshold value according to learning characteristics of the trainees. After the push prompt provides two processing methods of automatically correcting or forcing re-operation.

(5) To record the number and content of interactive operations such as requesting help and viewing data during the training process, the system classifies according to the trainees' learning characteristics, triggering automatic push help data, forcing to abort operation and reentering the two processing methods of explaining and learning mode according to the trigger threshold value.

\subsection{Adaptive Design in Test Assessment}

(1) Taking the parts identification test as an example, the parts are randomly disrupted, then the trainees match the parts with their corresponding names or functions or parameters. After the completion of the system, the system provides a positive-erroneous judgment and records. For the content with higher error rate in the test, according to the trainees' learning characteristics, the metadata value of the difficulty of the topic is dynamically adjusted, and the number of selected questions is increased in the random selection.

(2) Set up a wrong question bank to collect trainees' operating errors, violations, or unsafe operations, classify them according to the trainees' learning characteristics, dynamically adjust the misoperation deduction strategy of this step, and dynamically adjust the repeated operation threshold after the wrong operation of that step.

\section{Implementation of Adaptive Virtual Maintenance Training System}

\subsection{Virtual Interaction}

The virtual interaction method is divided into handle entity interaction and Ray interaction. Contact: When the button (option) is touched, the outer outline of the button (option) will light up in red, and the button (option) will appear larger than the other button (option). This means that the button (option) is already in contact with the device. Trigger: After touching, press the trigger button to represent the click on the button. After clicking, the button realizes its own function. As shown in Figure 2.

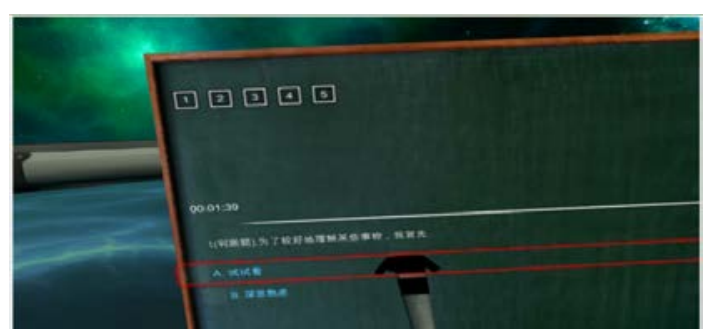

Fig. 2 virtual interaction in test process 


\subsection{Study Style Test}

When the learning style test is conducted, the system first sends the test scale information of the style test to the Unity engine, the Unity engine loads the test scale information, and then the Unity engine feeds back the user's test results to the training system. The test results are judged by the training system, and the user's learning style is finally obtained, and the style is then fed back to the Unity engine for output display. As shown in Figure 3.

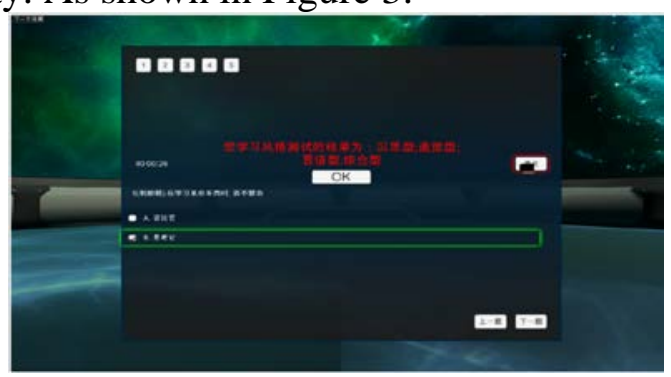

Fig. 3 the results of the learning style test

\subsection{The Realization of Push Training Content}

The training system judges according to the learning style, and finally obtains the push rule that conforms to the style, and then loads the push training content according to the rule. The system stipulates that each user will only perform a learning style test once. The system will record the test results under the user information. After re-entering the system, it will push out the training content that matches the user according to the previous test style. The system records the training of each user's push training content. When the system enters the system training again, the system will automatically read the last record and continue the training. In addition, there will be a corresponding test after the training content training several knowledge points. Only after the test standards are reached can the next knowledge point be trained. When training new training content, the system is automatically loaded for training. The training content that can be manually selected for training can only be the training content that has been trained. Untrained training content can not be manually selected. And there will be a corresponding record of training progress after each training content of the push list. As shown in Figure 4.

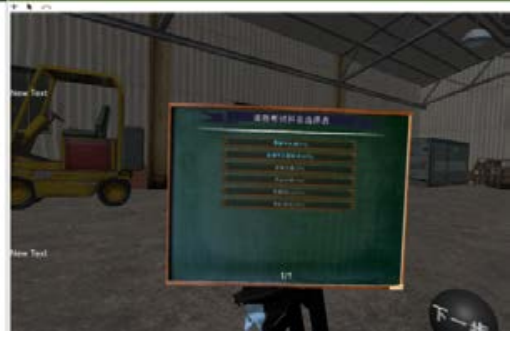

Fig. 4 Training list for pushing training content

\section{Summary}

In this thesis, an adaptive virtual maintenance training system based on cognitive mechanism has been studied. The adaptive virtual maintenance training system has been designed and studied respectively. Finally, the adaptive virtual maintenance training based on cognitive mechanism has been realized through the design of the virtual maintenance training system software. 


\section{References}

[1] Li Xingxin, Hao Jianping, Ye Fei, Zhao Zhengfan. The study of base problems and technique architecture of Virtual Maintenance Training. Microcomputer Information, 2010, (16) :30-32.

[2] Su Qunxing, Liu Pengyuan. Study of Virtual Maintenance Training System for Large Complex Equipment. Acta Armamentarii, 2006, (1): 79-83.

[3] Research on the Virtual Maintenance Training Platform Technology for Large Complex Equipment. Nanjing: Nanjing University of Science and Technology,2005.

[4] Wang Muxuan. Research and Implementation of Adaptive Learning System Based on Equipment Maintenance. Beijing: Beijing University of Posts and Telecommunications,2017

[5] Sui Guangyuan. An Experimental Reaserch on the Cognitive Law of Knowledge Points.Psychological Science. 2003,26(2):308-311. 